\title{
Greedy Path Planning for Maximizing Value of Information in Underwater Sensor Networks
}

\author{
Fahad Ahmad Khan, Saad Ahmad Khan, Damla Turgut and Ladislau Bölöni \\ Department of Electrical Engineering and Computer Science \\ University of Central Florida, Orlando, FL \\ Email: fahad.khan@ucf.edu, \{skhan, turgut, lboloni\}@eecs.ucf.edu
}

\begin{abstract}
Underwater sensor networks (UWSNs) face specific challenges due to the transmission properties in the underwater environment. Radio waves propagate only for short distances under water, and acoustic transmissions have limited data rate and relatively high latency. One of the possible solutions to these challenges involves the use of autonomous underwater vehicles (AUVs) to visit and offload data from the individual sensor nodes. We consider an underwater sensor network visually monitoring an offshore oil platform for hazards such as oil spills from pipes and blowups. To each observation chunk (image or video) we attach a numerical value of information (VoI). This value monotonically decreases in time with a speeed which depends on the urgency of the captured data. An AUV visits different nodes along a specific path and collects data to be transmitted to the customer. Our objective is to develop path planners for the movement of the AUV which maximizes the total VoI collected. We consider three different path planners: the lawn mower path planner (LPP), the greedy planner (GPP) and the random planner (RPP). In a simulation study we compare the total VoI collected by these algorithms and show that the GPP outperforms the other two proposed algorithms on the studied scenarios.
\end{abstract}

\section{INTRODUCTION}

Underwater sensor networks (UWSNs) had emerged as subject of active research, with applications such as underwater real-time surveillance of sea ports, dockyards, offshore drilling platforms and cultural heritage sites, environmental monitoring (for oil spills or carbon dioxide leaks) and studies of marine life. UWSNs pose challenges not encountered in other scenarios. The physical deployment and maintenance of the nodes is difficult, and sensors can be hindered by cloudy water and debris. However, one of the most difficult challenges pertain to underwater communication. While most traditional sensor networks use wireless communication, radio waves travel only over very short distances under water, as water absorbs large part of the electromagnetic spectrum. To overcome this limitation many UWSNs use acoustic communication. While acoustic signals can travel long distances underwater, they have relatively high latency (due to the speed of sound in water) and low bandwidht (values of 1-10 kbps are typical for distances of hundreds of meters). While such bandwidths are sufficient for transmitting compact sensing data, such as temperature readings, they are insufficient for video transmission and marginal for any any kind of image data.
One possible solution to this problem is to combine the underwater sensing nodes anchored to the sea floor with mobile units such as autonomous underwater vehicles (AUVs) which visit the sensor nodes, offload data using either short distance optical communication or physical connection, and then physically transport this data either to a collection point, or to the surface where it can be transmitted through open-air wireless transmission.

In this paper, we are considering a scenario where static underwater sensor nodes are equipped with cameras, data buffers and communication modules. The cameras record the environment of the node, and store the recordings as both image files and video files in a memory device (data buffer). An AUV is responsible for collecting this data from the sensor nodes using an optical communication technology which allows high data rates over short distances, which requires a physical visit by the AUV to the immediate neighborhood of the static node. After downloading the data from the sensor node, the AUV must travel to the surface to forward the data using open-air wireless technology to the sink. In addition to this both the AUV and the sensing nodes are equipped with acoustic communication modems. The data rate of the acoustic communication is too small to be useful in the transmission of images and video files. However, the acoustic communication allows the real-time exchange of synchronization messages and other meta data. For the scenario we are considering, the most important role of the acoustic communication is the transmission of beacon messages which indicate the presence of hot spots in certain areas of the sensor networks (see Figure 2). Hot spots are a regions of active interest for the user, which are either designated as such by the customer or self-detected by the sensor nodes. For instance, a hot spot for an offshore drilling platform might be an area where an oil spill is detected or suspected.

The objective of this paper is to propose and investigate various path-planning algorithms for the AUV. A path of the AUV includes the order and time-slots in which the AUV visits certain nodes to download the data and, respectively surfaces for the forwarding of the data to the base-station. The sequence of visits depends upon the goals of the user which determine the metrics and heuristics on the basis of which a path-planning algorithm can be formulated. The metric we use is Value of Information (VoI) associated with each data chunk. 
The VoI is a monotonically decreasing function in time and each data chunk has VoI function associated with it, which describes the initial VoI of the data as well as the way in which it descreases in time.

The remainder of this paper is organized as follows. In Section II we describe related work. In Section III we define a model for estimating the value of information of a data chunk. Section IV describes the deployment scenario we are considering. Section V formulates the AUV path planning problem for our scenario. Section VI discusses the algortihms we have used for path planning. Section VII describes the setup of our simulation experiments and their results. We conclude in Section VIII.

\section{RELATED WORK}

Path planning is a diverse subject and there is lot of literature on it specifically in the artificial intelligence community. A path planner essentially is an algorithm which discovers a valid route between two points on a map (usually represented by graph). Path planners are used to guide robotic/autonomic agents in their automated movement for path discovery and obstacle avoidance in an environment with a variety of constraints. Among the subgoals or constraints would be to find the most optimal route in terms of shortest distance, minimal time, low fuel expenditure, and so on. They essentially convert a set of high level specifications into low level instructions thus breaking down the problem solution into simpler steps.

There are generally two types of path planners: pregenerative and reactive [8]. If the path is planned before the AUV starts the course then the algorithm is pre-generative while if the plan is updated during the course in reaction to changes in objectives, constraints or obstacles then the algorithm is reactive. Maps represented by graphs are usually solved by planners which are variants of Dijkstras Algorithm. One of these well-known variants is $A^{*}$ which plans a path based on a set of heuristics provided.

AUVs are autonomous entities and hence require path planning techniques to help them navigate beneath seas and oceans. They have certain issues that affect them more heavily as compared to dry-land autonomous agents in terms of navigation (e.g. they suffer from inherent limitations of slow communication, limited sensing and power issues). But given the number of issues particular to underwater navigation and the existing research on path planning in general, AUV path planning is still a comparatively open research area.

An earlier path planning technique employed case-based reasoning in [14]. One of the first efforts to use $A^{*}$ for path planning for AUVs was by Carroll et al [4]. Garau et al. [7] used the potential field based methods and some of its drawbacks has been resolved by Fu-guang et al. [6]. Genetic Algorithms [11], [5], [1] and evolutionary algorithms [10] were also employed to present their niche on AUV path planning.

Turgut and Boloni [12] introduced an information quality metric on the basis of which a methodology was devised to retrieve data in a particular priority from different types of sensor networks. The information quality metric was VoI which essentially makes the user aware of the degradation/decay of the quality of information of data (pertaining to a set of sensed stimuli) with respect to time (since the time the stimuli were first recorded to the present instant). The VoI has been initially applied within the intruder tracking sensor networks [12], [13]. The transmission scheduling of sensor nodes via acoustic links to the sink as well as the path planning of an AUV to collect data from sensor nodes have been also explored [3], [2]. In either of the application domain, the goal was to maximize the quality of the gathered data.

\section{VALUE OF INFORMATION}

VoI is an intuitive metric for estimating the quality of an information set. The idea behind VoI can be described by a scenario where an actuation action has to be taken on the basis of sensed data. The sensed information is of more value at present as compared to it being processed for actuation at a later time. For instance, if an oil leak appears, the customer must try to take action to repair the leak at the earliest time. The more earlier the information (sensed by nodes) arrives to the user (sink and controller) the more swiftly the user will be able to take any decision for repairing and patching up the leaks.

The VoI is always monotonically decreases - an early arrival of the information never decreases its value. For many scenarios, the $\mathrm{VoI}$ can be described in terms of an exponential decay (although other forms are possible):

$$
F_{\text {VOI }}(t)=A e^{-B t}
$$

The value $A$ represents the initial value of the information this is the VoI which would be realized if the customer would receive the data chunk at the moment in which it had been sensed. Figure 1 shows three different profiles describing the fact that different data chunks can have different VoI profiles. For instance we can have a lower magnitude $A$ or a lower exponent multiplier $B$ or both. In case of an emergency we can have a greater magnitude of $A$ or exponent multiplier $B$ as compared to the normal operation. A higher magnitude of $A$ will represent information with a higher initial value while a higher value of $B$ represents a faster decay of the VoI - that is, an information which is more "urgent".

The two constants that give shape to these exponential curves are $A$ and $B$. Let us illustrate by example the use of these constants. Let us hypothetically declare a time $T_{\text {response }}$ which is hard limit on the activation of an actuation response to a sensed stimuli. If the response is initiated after time TB then there would be not much use of it as the required damage is already done, therefore, it is imperative that the information be delivered from the sensor nodes to the sink and controller within the time TB so the correct response could be initiated at the earliest. Let us suppose there are two events $E_{1}$ and $E_{2}$. This then implies that the response to event $E_{1}$ should be more swift as chances are that the validity of an actuation response might expire quickly. For the event $E_{1}$ a lower value 


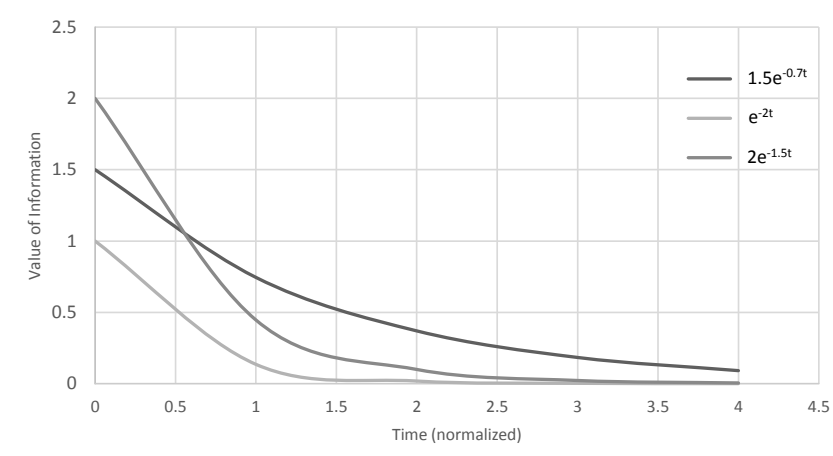

Fig. 1. VoI for different decay times

of $B$ could be chosen indicating a quicker decay and hence demanding an urgent action.

$$
T_{\text {response E1 }}>T_{\text {response } E 2} \Rightarrow B_{E 1}<B_{E 2}
$$

Let the urgency of an event be described by the magnitude $M_{\text {critical }}$. Now suppose events $E_{1}$ and $E_{2}$ have the same $T_{\text {response }}$ but event $E_{1}$ demands more urgent action e.g. an oil spill. Then we can set the magnitude $A_{1}$ of event $E_{1}$ to be of a higher value as compared to $A_{2}$ for event $E_{2}$, thereby, laying a higher emphasis on the importance of the information it has so as to demand a suitable actuation response more urgently.

$$
M_{\text {critical E1 }}>M_{\text {critical E2 }} \Rightarrow A_{E 1}>A_{E 2}
$$

\section{Deployment Scenario}

We are considering the case where there are underwater sensor nodes arranged in a grid (see Figure 2). The nodes have the ability to sense and classify data into two distinct categories. Class $C_{\text {Norm }}$ has data associated with it which is reported to be normal and each data bundle in this class has a VoI profile defined by the function $f_{\text {VoI Norm }}(t)$. This function is common to all the members in the class (i.e. the constants take on the same values $A_{1}$ and $B_{1}$ for each data bundle). While in case of an emergency the data is classified to be part of class $C_{\mathrm{Hot}} \mathrm{Spot}$ and the function for members

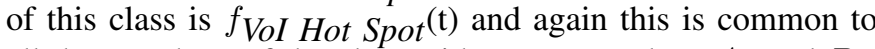
all the members of the class with constant values $A_{2}$ and $B_{2}$.

The AUV goes about collecting data from various nodes in a way that it is desirable to maximize the value of information required. The AUV can resurface at any point during collection of data and can communicate using wireless medium to transfer the accumulated data up till that instant to a basestation. Once it transmits all the data it returns to resume offloading data from the underwater sensor nodes.

Suppose there is a senor node that has detected a critical event and classifies it to be in $C_{H o t}$ Spot . It will then raise an alarm by signaling a beacon through an acoustic channel. Once the AUV detects the beacon it will re-evaluate its strategy of node visitation sequence and will devise a way to quickly reach the node that raised the alarm. The region which the AUV has to visit to offload this critical data is referred to as Region of Interest (RoI) or Hot Spot (HS). While on its way to the Hot Spot the AUV can devise strategies to gather data from nodes it passes by on its way. It can have other strategies and the agenda is to discuss these strategies which we will refer to as "heuristics for path planning".

\section{AUV Path Planning Problem}

The problem for the path planning of the AUV is to maximize the value of information, collected in the form of data chunks from a set of nodes. Therefore, an AUV needs to plan a priority path for visiting the nodes for data collection. The sensing nodes attribute a value, decaying in time, with each chunk of sensed data. For urgent data, the decay for value of information is high whereas its low otherwise.

Therefore, we can mathematically represent the problem for VoI path planning as the $(\mathbf{L}, \mathbf{H}, \mathbf{A}, \mathbf{V}(\mathbf{B}, \mathbf{t}))$ where

- $H \subset S$, is a set of nodes with urgent VoI profiles, i.e., having maximum value of information and is given as

$$
V(B, t) \forall x \in \mathbf{H}>V(B, t) \forall x \in(\mathbf{S N}-\mathbf{H})
$$

where $x$ is a sensor node

- the value of information $V(B, t)$ when AUV collects data from node at time $t$

- $\mathbf{L}$ is a finite set of locations of the high profile $\mathrm{VoI}$ sensor nodes

- the action $A$ for selecting the next visiting node from the list of nodes having urgent VoI profiles, i.e., $\mathbf{L}$

The goal for the AUV is to use a path planning policy for creating a priority list of nodes $\pi$ to be visited, to maximize the value of information $\sum_{i=1}^{t} V(B, i)$ during its detour for collecting the data packets.

\section{Path Planning Algorithms}

In the following, we discuss the three algorithms that we consider for the path planning problem.

\section{A. PathPlanner 1: Lawn mover path planner (LPP)}

The Lawn mover path planner (LPP) is an information maximizing path planner which provides the maximum amount of VoI for a homogeneous VoI profile throughout the sensor network. For a homogeneous profile all the sensor nodes will have same magnitude and decay time for the VoI. An example for a homogeneous profile can be a set of few neighbor nodes observing the oil spill over a large region where each node has the information of same magnitude and importance. In this scenario, besides collecting other information, the AUV still needs to visit every node to collect additional information that would be of importance to evaluate the oil spill. LPP has a practical significance in the absence of hot-spot as it provides the best greedy path planning for a homogeneous profile network.

Algorithm 1 highlights the different steps involved for an AUV to plan its path using LPP. The AUV maintains a history of nodes that it has already visited besides maintaining a priority list of neighbor nodes. The priority list of a node schedules the next neighbor nodes among the other neighbors in which the AUV must visit. For our implementation, we 


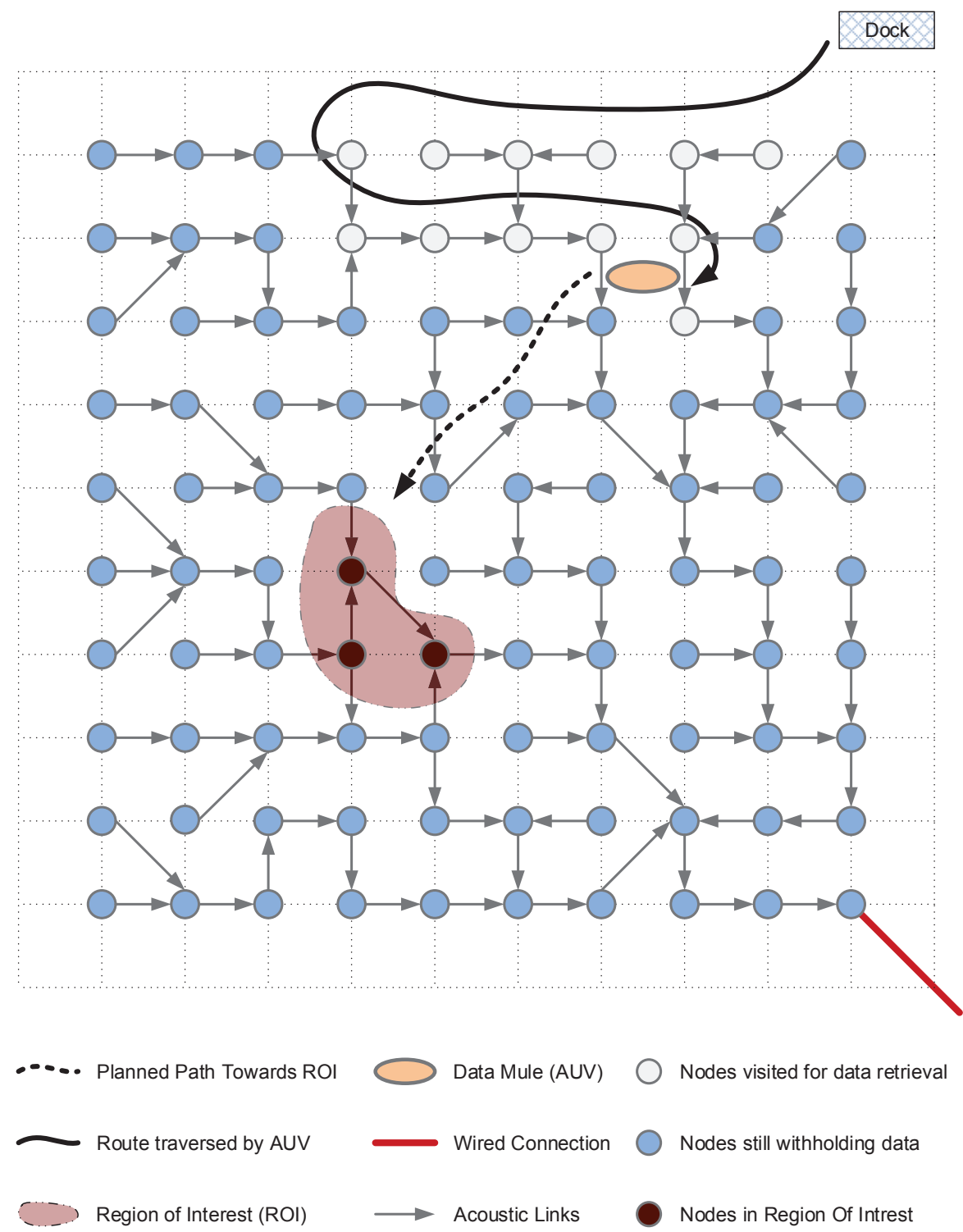

Fig. 2. The deployment scenario

prioritize the selection of the next neighbor which is located on the East of current node, then West, followed by South and finally North.

\section{B. PathPlanner 2: Greedy path planner (GPP)}

The Greedy path planner (GPP) provides path to next destination to maximize information in a heterogeneous VoI profile network. The idea is to maintain the overall VoI profile of the network and visit the node of utmost importance. Therefore, in the absence of hotspots, GPP is scheduled to act as an LPP. This means that GPP would proactively follow the schedule of an LPP but would reactively follow a greedy approach for reaching a hotspot. In Algorithm 2, we can see that the AUV maintains the list of VoI profiles for different hotspots in the network. Afterwards, it schedules the destination list giving priority to the hotspot with maximum VoI profile.

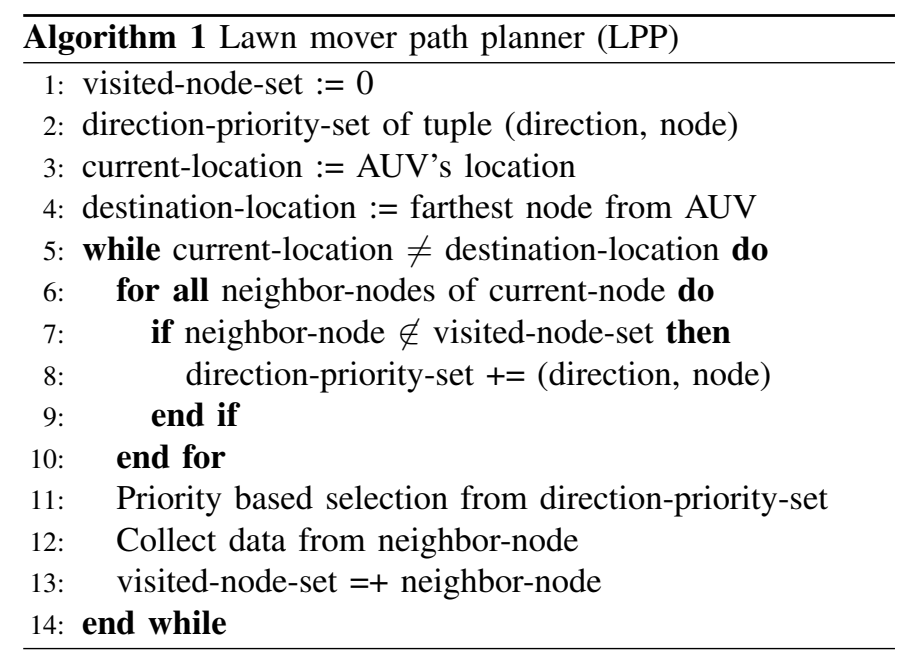




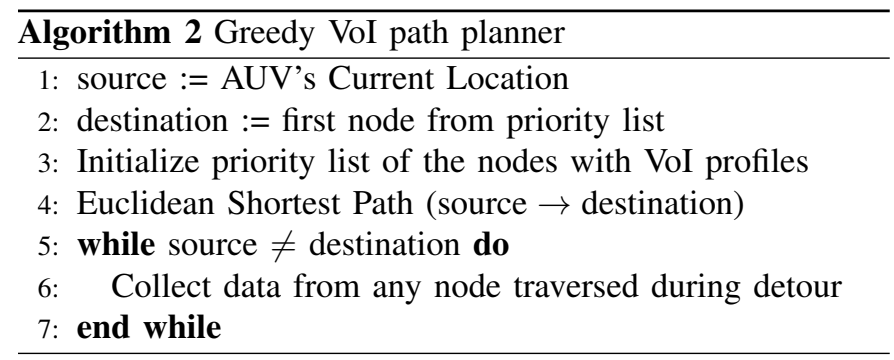

TABLE I

EXPERIMENTAL PARAMETERS

\begin{tabular}{|l|l|}
\hline \multicolumn{1}{|c|}{ Parameter } & Values \\
\hline \multicolumn{2}{|c|}{ General properties of ROI } \\
\hline ROI size & $1000 \times 1000 \mathrm{~m}$ \\
Node deployment & uniform grid \\
Number of sensor nodes & 100 \\
Transmission range & $120-140 \mathrm{~m}$ \\
Sensing range & $70 \mathrm{~m}$ \\
Mobile sink speed & $1-20$ knot \\
Experiment length & 500 time-steps \\
VoI Profile & $\{10,0.04\}$ \\
\hline \multicolumn{2}{|c|}{ Other Parameters } \\
\hline Heuristics & LPP, GPP, RPP \\
Number of hotSpots & $0-5$ \\
Location of hotspots & Random \\
Decay-time of VoI for hotspots & $0.4-0.7$ \\
\hline
\end{tabular}

\section{PathPlanner 3: Random path planner (RPP)}

For evaluation purposes, we implemented a third path planner, a random path planner (RPP) in which the AUV randomly chooses the next sensor node for data collection. The detours towards the selected node uses the Euclidean shortest path planner. This path planner can be thought of as a noisy planner which is incapable of scheduling proper visits to nearest nodes or hotspots due to inconsistency of evaluation of VoI data-profiles (which maybe due to intruder noise in the system). Hence, the AUV schedules various path visits to different sensor nodes irrespective of the critical nature of the VoI in the underlying sensor networks.

\section{SimulationStudy}

We consider an underwater sensor network of 100 nodes deployed in a uniform grid over $1 \times 1 \mathrm{~km}^{2}$ area as shown in Figure 2. These sensor nodes have an acoustic communication medium that allows them to send the data to the AUV. We consider the AUV, a Katrina boat [9] that can speed upto 10 knots in rough water. The travel time of AUV varies between two sensor nodes with the speed of wind gushes and roughness of water surface. For our experiments, we ignore these two environmental factors and consider a calm water profile. For collecting the data, the AUV can connect with the sensing node with a data rate of $10 \mathrm{Mbps}$ using the optical links or can use acoustic links for collecting the data packets.

In order to determine the value profiles of the data chunks, we divide them into various observation classes over the span of simulation. Each of the value of information profile has a different urgency and basis information levels.

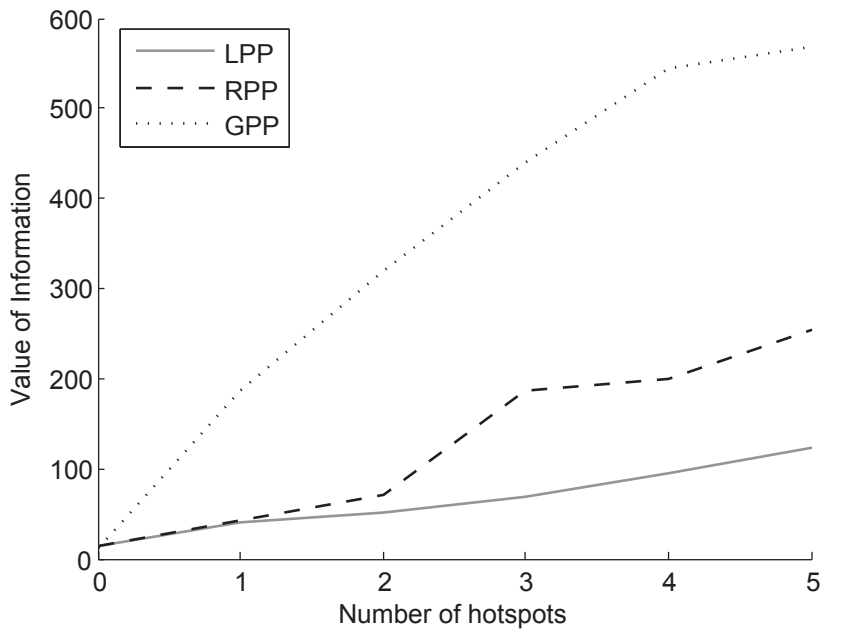

Fig. 3. The aggregated collected value of Information over time-span

We evaluate the effect of collected value of information with the increasing number of hotspots in the region of interest. For this scenario, we randomly generate different locations of hotspots having random decay time for VoI but with same magnitude of VoI. For each simulation, the number of hotspots remain fixed, hence, we vary the number of hotspots from one to five at randomly selected locations. From the results in Figure 3, we can see that the magnitude difference of collected VoI between GPP and LPP increases with the increase in the number of hotspots. This shows that GPP outperforms LPP with the increase in value of information of the overall network.

For comparing the efficiency of different path planners over the course of AUV's detour we compare the collected value of information using each planner at different time steps. Figure 4 shows the time series output for different path planners. For this scenario, we considered three randomly chosen hotspots which had to be covered during the AUV's detour for collecting data packages. From Figure 4, we can observe that greedy path planner was able to efficiently schedule visits to all three hotspots as compared to the rest of the two path planners.

Figure 5 shows us the total collected value of information over the span of simulation. The steps in the staircase graph indicates the time at which the AUV was able to gather the maximum the value of information from the hotspots. It's evident that greedy path planners outperform rest of the two path planners for maximizing the collected value of information.

Figure 6 shows the confidence intervals for VoI ratio collected by the AUVs with the increasing number of hotspots during each simulation. We can observe that for an LPP, the ratio remains constant most of the time because LPP does not takes into consideration the locations of hotspots for path planning. We also observe that the performance of an RPP increases because the probability of visiting hotspots increases with an increasing number of hotspot locations in the network. 


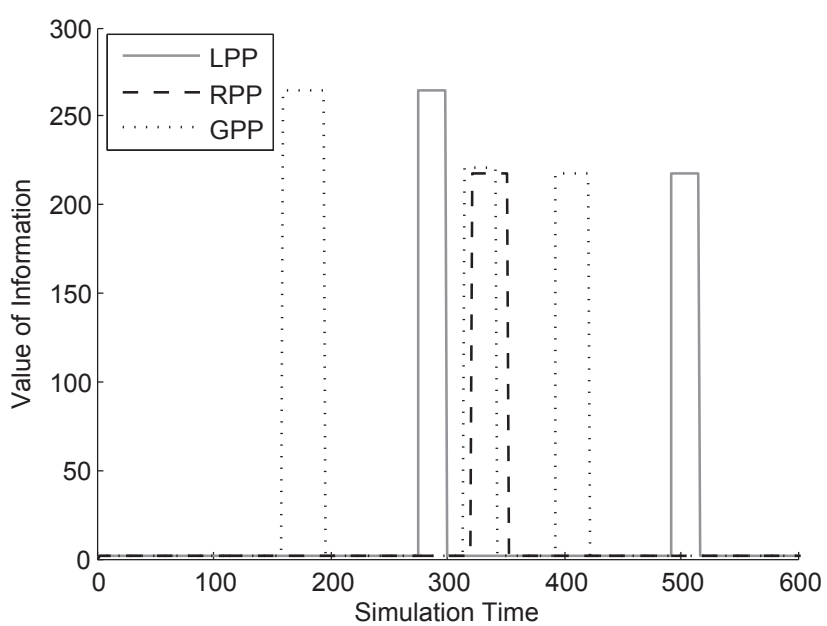

Fig. 4. Time Series Graph for Collected Value of Information

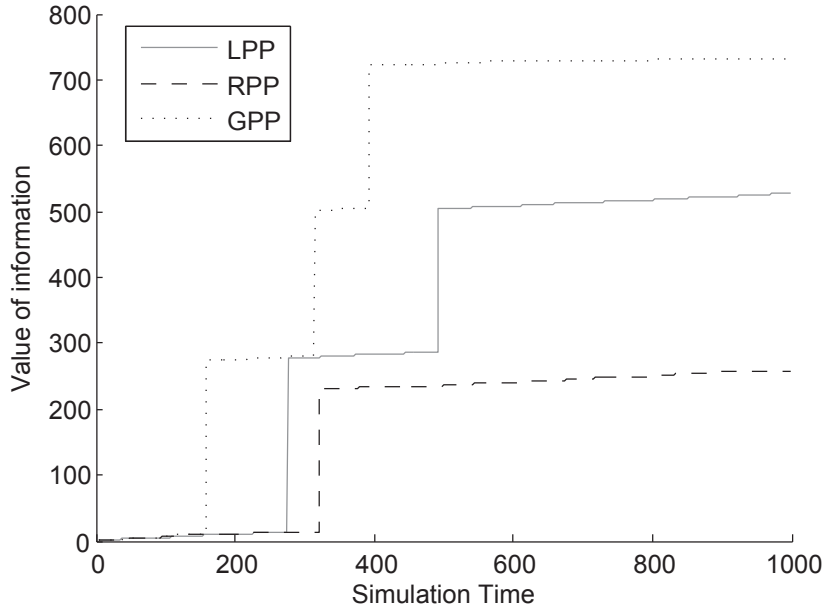

Fig. 5. Time Series Graph for the aggregated Value of Information over the span of AUV's detour

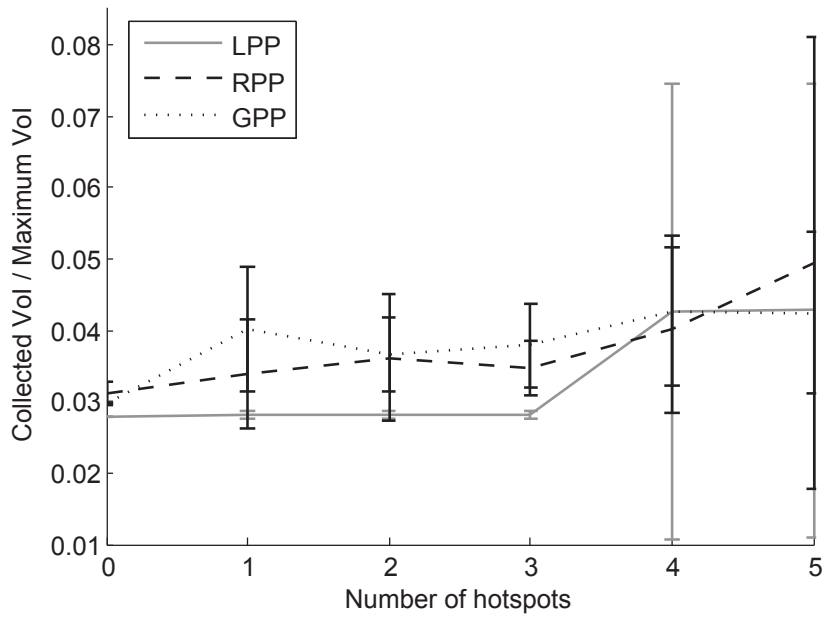

Fig. 6. The confidence interval for VoI ratio

\section{CONCLUSION}

In this paper, we proposed path-planning algorithms for an AUV which acts as a mobile sink node for the underwater sensor nodes. We used a heuristic called Value-of-Information (VoI) as the metric for the path planning. The VoI is a strictly monotonically decreasing function associated with each data bundle and serves as a marker for decay of the quality of information with respect to time of that data bundle. We consider the scenario having sensing regions with different VoI profiles for different data bundles. The objective of this paper was to analyze various path planners for an AUV to maximize the collected value of information by the AUV. The results show that VoI greedy path planner outperforms others.

\section{REFERENCES}

[1] A. Alvarez, A. Caiti, and R. Onken. Evolutionary path planning for autonomous underwater vehicles in a variable ocean. IEEE Journal of Oceanic Engineering, 29(2):418-429, 2004.

[2] S. Basagni, L. Bölöni, P. Gjanci, C. Petrioli, C. Phillips, and D. Turgut. Maximizing the value of sensed information in underwater wireless sensor networks via an autonomous underwater vehicle. In IEEE INFOCOM'14, pages 988-996, April-May 2014.

[3] L. Bölöni, D. Turgut, S. Basagni, and C. Petrioli. Scheduling data transmissions of underwater sensor nodes for maximizing value of information. In IEEE GLOBECOM, pages 438-444, December 2013.

[4] K. P. Carroll, S. R. McClaran, E. L. Nelson, D. M. Barnett, D. K. Friesen, and G. William. AUV path planning: an $\mathrm{A}^{*}$ approach to path planning with consideration of variable vehicle speeds and multiple, overlapping, time-dependent exclusion zones. In Proc. of IEEE Symposium on Autonomous Underwater Vehicle Technology, pages 79-84, 1992.

[5] R. Fox, A. Garcia, and M. Nelson. A three-dimensional path planning algorithm for autonomous vehicles. In Proc. of Int'l Symposium on Unmaned Untethered Submersible Technology, pages 546-553. University of New Hampshire - Marine Systems, 1999.

[6] D. Fu-guang, J. Peng, B. Xin-qian, and W. Hong-Jian. AUV local path planning based on virtual potential field. In Proc. of IEEE Int'l Conf. of Mechatronics and Automation, volume 4, pages 1711-1716, 2005.

[7] B. Garau, A. Alvarez, and G. Oliver. Path planning of autonomous underwater vehicles in current fields with complex spatial variability: an A* approach. In Proc. of IEEE Int'l Conf. on Robotics and Automation (ICRA), pages 194-198, 2005.

[8] Y. K. Hwang and N. Ahuja. Gross motion planning A Survey. ACM Computing Surveys (CSUR), 24(3):219-291, 1992.

[9] MITSG. Katrina boat: A diesel electric vertical profiling autonomous surface craft. MITSG, 2010.

[10] D. Rathbun, S. Kragelund, A. Pongpunwattana, and B. Capozzi. An evolution based path planning algorithm for autonomous motion of a UAV through uncertain environments. In 21st Proc. of IEEE Conf. of Digital Avionics Systems, volume 2, pages 8D2-1-8D2-12, 2002.

[11] K. Sugihara and J. Yuh. GA-based motion planning for underwater robotic vehicles. In Int'l Symposium on Unmanned Untethered Submersible Technology, pages 406-415, 1997.

[12] D. Turgut and L. Bölöni. A pragmatic value-of-information approach for intruder tracking sensor networks. In Proc. of the IEEE ICC'12, pages 4931-4936, June 2012.

[13] D. Turgut and L. Bölöni. IVE: improving the value of information in energy-constrained intruder tracking sensor networks. In Proc. of IEEE ICC'13, pages 6360-6364, June 2013.

[14] C. Vasudevan and K. Ganesan. Case-based path planning for autonomous underwater vehicles. Autonomous Robots, 3(2-3):79-89, 1996 